\title{
Computing the exponent of a Lebesgue space
}

\author{
TIMOTHY H. MCNICHOLL
}

\begin{abstract}
We consider the question as to whether the exponent of a computably presentable Lebesgue space whose dimension is at least 2 must be computable. We show this very natural conjecture is true when the exponent is at least 2 or when the space is finite-dimensional. However, we also show there is no uniform solution even when given upper and lower bounds on the exponent. The proof of this result leads to some basic results on the effective theory of stable random variables.
\end{abstract}

2010 Mathematics Subject Classification 03D78 (primary); 03D45 (secondary)

Keywords: computable analysis, computable structure theory, computable probability theory, functional analysis

\section{Introduction}

Computable structure theory studies computable presentations of mathematical structures and their relationship to each other. These investigations support the advancement of computable model theory and effective mathematics and the theory of computation generally. Roughly speaking, a computable presentation of a structure is a way of defining computation on the structure. In the case of countable structures, this is achieved by numbering the elements of the structure in a suitable way; namely so that the induced relations and operations on the natural numbers are computable. Recently, the field has expanded its purview by investigating metric structures such as metric spaces and Banach spaces (see eg Melnikov [19], Melnikov and Nies [21], Melnikov and Ng [20], McNicholl [17], Clanin, McNicholl and Stull [6], and Brown and McNicholl [3]). In the case of Banach spaces, a computable presentation is a numbering of a linearly dense sequence in such a way that the norm and the vector space operations can be computed. A formal definition is given in Section 2 below.

Computable presentations of Banach spaces have been studied at least since the seminal monograph of Pour-El and Richards [23]. In that text, and in subsequent developments, much attention is paid to computing on Lebesgue spaces; that is, $L^{p}$ spaces for some value of $p$ (see eg Zhong and Zhang [29] and Kunkle [13]). This focus makes sense 
since such spaces are of fundamental importance for analysis and applied mathematics. It is usually assumed that the exponent of the space is computable. This is a natural assumption; in fact, one might even consider the specification of the exponent to be part of the presentation. Using the classification of separable $L^{p}$ spaces (see eg Cembranos and Mendoza [5]) it is fairly easy to show that when $p$ is computable, every separable $L^{p}$ space has a computable presentation.

Here, we wish to take a step back and question the necessity of assuming the computability of the exponent. That is, we consider the question: "If a Lebesgue space is computably presentable, does it follow that its exponent is computable?" Stated this way, the answer is easily seen to be 'no'. For, no matter the exponent, a one-dimensional Lebesgue space is just the field of scalars. So, we restrict our attention to spaces of dimension at least 2. Thus, we ask: "If a Lebesgue space whose dimension is at least 2 is computably presentable, does it follow that its exponent is computable?"

A partial answer to this question is given by Brown, McNicholl and Melnikov in [4]. Namely, it is shown that if a Lebesgue space is computably presentable then its exponent is right-c.e. if it is smaller then 2, otherwise it is left-c.e. Here, we strengthen this result by showing that the exponent must be computable if it is larger than 2 or if the space is finite-dimensional.

While we do not have a complete answer to our question, we nevertheless present some strong evidence that the answer is likely to be 'no'. Namely, we show that even given rational upper and lower bounds on the exponent as advice, there is no uniform procedure for computing the exponent of a Lebesgue space from an index of one of its presentations.

In part, the motivation for trying to answer this question is that, while it is somewhat technical, it nevertheless is a fundamental question to consider for the theory of computing on Banach spaces. In particular, it would be very surprising to find one could compute on a Lebesgue space without knowing its exponent, for then even the norm would seem to be out of reach. But, the larger part of the motivation is the number of interesting connections that are made between computability and a broad swath of material in functional analysis and probability. For example, the proof of the aforementioned result by Brown, McNicholl and Melnikov utilizes the modulus of convexity for a strictly convex Banach space and a method due to O. Hanner for computing this modulus for a Lebesgue space from its exponent [10]. The proof of the result presented here for exponents larger than 2 makes use of a syntactic characterization due to J. L. Krivine [12] of the Banach spaces that contain a copy of an $L^{p}$ space, as well as some non-embedding results due to Banach [1] and Paley [22]. The non-uniformity 
result will lead us to consider effective aspects of the theory of stable random variables in probability theory. This is for the sake of proving an effective version of an embedding result due to Bretagnolle, Dacunha-Castelle and Krivine [2].

The paper is organized as follows. Section 2 summarizes background information from probability, functional analysis, and computable analysis. Section 3 contains some preliminary results from computable analysis. The result for exponents larger than 2 is then presented in Section 4. In Section 5, we leave the main track of our thought to prove the results we require from the effective theory of stable random variables. In Section 6, we demonstrate the effective version of the embedding result just mentioned, and then in Section 7, we demonstrate the non-uniformity result. Finally, the proof of the result for finite-dimensional spaces is given in Section 8. In the conclusion, we summarize the results again and state a conjecture.

\section{Background}

\subsection{Background from functional analysis}

Let $\mathbb{F}$ denote the field of scalars, which can be $\mathbb{R}$ or $\mathbb{C}$. Let $\mathbb{F}_{\mathbb{Q}}=\mathbb{F} \cap \mathbb{Q}(i)$. That is, $\mathbb{F}_{\mathbb{Q}}$ is the field of rational scalars.

Most of our arguments are not affected by the choice of scalars. When the field of scalars is of concern, we use the notations $L^{p}(\Omega ; \mathbb{R})$ and $L^{p}(\Omega ; \mathbb{C})$ for the real and complex $L^{p}$ spaces over the measure space $\Omega$.

The following was introduced in McNicholl [18] and will later be used to build isometric embeddings of $L^{p}$ spaces.

Definition 2.1 Suppose $1 \leq p<\infty, \mathcal{B}$ is a Banach space, and $v_{1}, \ldots, v_{n} \in \mathcal{B}$. We say $v_{1}, \ldots, v_{n}$ are $L^{p}$-formally disjointly supported if

$$
\left\|\sum_{j} \alpha_{j} v_{j}\right\|_{\mathcal{B}}^{p}=\sum_{j}\left|\alpha_{j}\right|^{p}\left\|v_{j}\right\|_{\mathcal{B}}^{p}
$$

for all scalars $\alpha_{1}, \ldots, \alpha_{n}$. We say that a sequence $\left\{v_{n}\right\}_{n \in \mathbb{N}}$ of vectors is $L^{p}$-formally disjointly supported if $v_{0}, \ldots, v_{M}$ are $L^{p}$-formally disjointly supported for all $M$.

The choice of terminology in Definition 2.1 is motivated by the following two facts. First, if $f_{1}, \ldots, f_{n} \in L^{p}(\Omega)$ are disjointly supported, then they are $L^{p}$-formally disjointly 
supported. By a result of J. Lamperti, if $p \neq 2$ then $L^{p}$-formally disjointly supported vectors in $L^{p}(\Omega)$ are disjointly supported [14].

Our computation of exponents larger than 2 utilizes the following two theorems. The first is a result on non-embeddings, and the second is a syntactic characterization of spaces containing a copy of a Lebesgue space.

Theorem 2.2 Suppose $2<p_{1}<p_{2}<\infty$.

(1) No infinite-dimensional $L^{p_{2}}$ space isometrically embeds into an $L^{p_{1}}$ space.

(2) No infinite-dimensional $L^{p_{1}}$ space isometrically embeds into an $L^{p_{2}}$ space.

Theorem 2.3 Suppose $1 \leq p<\infty$, and let $\mathcal{B}$ be a Banach space. Let $r, s$ be positive integers so that $2(r-1)<p<2 r \leq 4 s$. Then, $\mathcal{B}$ isometrically embeds into an $L^{p}$ space if and only if for all $v_{1}, \ldots, v_{n} \in \mathcal{B}$ and all $\alpha_{1}, \ldots, \alpha_{n} \in \mathbb{R}$,

$$
(-1)^{r} \sum_{\sigma \in\{-1,1\}^{2 s}} \sum_{\tau \in\{1, \ldots, n\}^{2 s}}\left\|\sum_{j<2 s} \sigma(j) v_{\tau(j)}\right\|_{\mathcal{B}}^{q} \cdot \prod_{j<2 s} \alpha_{\tau(j)} \geq 0
$$

provided $\sum_{j} \alpha_{j}=1$.

Part (1) of Theorem 2.2 appears in Banach's seminal 1932 monograph [1]. Part (2) of Theorem 2.2 was proven by Paley in 1936 [22]. Theorem 2.3 was proven by J.L. Krivine in 1965 [12].

\subsection{Background from probability: stable random variables}

The theory of stable random variables was initiated by P. Levy in 1925 [15] and further formalized in his 1951 monograph [16]. Applications of stable random variables actually began in 1919 with Holtsmark's work in astronomy [11]. Afterward, they were been applied to many fields including physics, biology, economics, finance, and signal processing (see eg Samorodnitsky and Taqqu [24] and Zolotarev [30] for surveys). Today stable distributions continue to be a very active area of investigation in both pure and applied probability theory; see eg Uchiyama [25], Xu [27], and $\mathrm{Yu}, \mathrm{Li}$ and Zhang [28].

The material in this subsection is drawn from [24] and [30].

The following is a consequence of the proof of Theorem 1.2.2 of Durrett [7] and will be used to generate random variables from distribution functions. 
Proposition 2.4 If $F$ is a cumulative distribution function, and if $g(t)=\sup F^{-1}[(0, t)]$ for all $t \in(0,1)$, then $F$ is the distribution function of $g$.

We write $X={ }_{d} Y$ when $X$ and $Y$ are identically distributed random variables.

A random variable $X$ is said to be symmetric if $X={ }_{d}-X$.

Definition 2.5 Let $X$ be a random variable.

(1) $X$ is said to be stable if for all positive $A, B$, there is a positive $C$ and a real $D$ so that whenever $X_{1}$ and $X_{2}$ are independent random variables and $X_{1}={ }_{d} X_{2}={ }_{d} X$, $A X_{1}+B X_{2}={ }_{d} C X+D$.

(2) If, in addition, we can always choose $D$ to be zero, then $X$ is strictly stable.

The property of stability is a property of the distribution of a random variable. Thus, we shall also speak of stable distributions. Every stable distribution is characterized by four parameters. The first of these is the stability index whose existence is given by the following theorem which is proved in Section VI.1 of Feller [9].

Theorem 2.6 Suppose $X$ is stable. Then there is a unique real number $r \in(0,2]$ so that whenever $A, B>0$ and $X_{1}, X_{2}$ are independent copies of $X$ (ie $X_{1}={ }_{d} X_{2}={ }_{d} X$ ), $A X_{1}+B X_{2}={ }_{d}\left(A^{r}+B^{r}\right)^{1 / r} X+D$ for some $D \in \mathbb{R}$.

Definition 2.7 If $X$ is stable, then the number $r$ in Theorem 2.6 is called the index of stability of $X$. We also say $X$ is $\alpha$-stable.

It is well known that the 2-stable random variables are precisely the Gaussian random variables and that the 1 -stable random variables are precisely the Cauchy random variables. The aforementioned distribution of Holtsmark is 3/2-stable.

When $X$ is a random variable, let $\phi_{X}$ denote the characteristic function of $X$; that is $\phi_{X}(t)=E\left[e^{i X t}\right]$. Recall that two random variables are identically distributed if and only if they have the same characteristic function. Characteristic functions are the main tools for the analysis of stable random variables. In particular, the form of the characteristic function of a stable random variable leads to the remaining three parameters that characterize a stable distribution. This is the content of the next theorem.

Theorem 2.8 Let $X$ be a random variable.

(1) If $X$ is $r$-stable, then there exist unique $\sigma \geq 0, \beta \in[-1,1]$, and $\delta \in \mathbb{R}$ so that:

$$
\phi_{X}(t)=\left\{\begin{array}{cc}
\exp \left(-\sigma^{r}|t|^{r}\left(1-i \beta\left(\operatorname{sgn}(t) \tan \left(\frac{\pi r}{2}\right)\right)+i \delta t\right)\right) & r \neq 1 \\
\exp \left(-\sigma|t|\left(1+i \beta \frac{2}{\pi} \operatorname{sgn}(t) \ln |t|\right)+i \delta t\right) & r=1
\end{array}\right.
$$


(2) If $\Phi_{X}$ has the form in Equation (1), then $X$ is $r$-stable.

The numbers $\sigma, \delta$, and $\beta$ are known as the scale, shift, and skewness parameters of $X$ as well as its distribution. We note that a stable random variable is symmetric if and only if its skewness and shift parameters are 0 . In addition, a 1 -stable random variable is strictly stable if and only if its skewness parameter is 0 . On the other hand, when $r \neq 1$, an $r$-stable random variable is strictly stable if and only if its shift parameter is 0 .

For the sake of computing moments, we will need to know the asymptotic behavior of the tail distributions of stable random variables. These are given by the following theorem.

Theorem 2.9 Suppose $X$ is an $r$-stable random variable, and suppose $\sigma, \beta$, and $\delta$ are its scale, skewness, and location parameters respectively. Then, there is a positive number $C$ so that the following hold for all sufficiently large $x$.

(1) If $r=2$, then:

$$
P[X<-x]=P[X>x] \leq \frac{C}{x} \exp \left(-x^{2} /\left(4 \sigma^{2}\right)\right)
$$

(2) If $r<2$, then $\max \{P[X>x], P[X<-x]\}<C x^{-r}$.

Part (1) is proven in Feller [8]. Part (2) follows from Property 1.2.15 of [24].

We will also use complex-valued stable random variables. The definition of stability for these random variables is a straightforward adaptation of the definition for the real case.

Definition 2.10 Suppose $X$ is a complex-valued random variable.

(1) $X$ is stable if for all $A, B>0$ there exist a $C>0$ and a $D \in \mathbb{C}$ so that whenever $X_{1}$ and $X_{2}$ are independent copies of $X, A X_{1}+B X_{2}={ }_{d} C X+D$.

(2) If we can always choose $D=0$, then we say $X$ is strictly stable.

Complex random variables also posses indices of stability.

Theorem 2.11 Suppose $X$ is a stable complex-valued random variable. Then, there is a unique $r \in(0,2]$ so that for all $A, B>0$, there is a $D \in \mathbb{C}$ so that whenever $X_{1}$ and $X_{2}$ are independent copies of $X, A X_{1}+B X_{2}={ }_{d}\left(A^{r}+B^{r}\right)^{1 / r}+D$.

Again, the number $r$ is called the index of stability of $X$.

When dealing with complex-valued random variables, we will need a stronger property than symmetry. 
Definition 2.12 A complex-valued random variable $X$ is isotropic if $\zeta X={ }_{d} X$ for every unimodular complex number $\zeta$.

The characteristic function of a complex-valued random variable $X$ is defined to be $\phi_{X}(z)=E[i\langle X, z\rangle]$ (where $\langle$,$\rangle is the standard inner product on \mathbb{R}^{2}$ ). Again, these functions characterize the distribution of $X$. Their forms are known, but these results will not play any role in our investigations. The interested reader is referred to Chapter 2 of [24].

\subsection{Background from computable analysis}

Here we present the formal definitions of presentation and computable presentation of a Banach space. We also define the computable vectors and sequences of a presentation and the computable maps between presentations. Our approach is essentially that in Pour-El and Richards [23]. See also Weihrauch [26].

Let $\mathcal{B}$ be a Banach space. A presentation of $\mathcal{B}$ is a pair $\left(\mathcal{B},\left\{v_{n}\right\}_{n \in \mathbb{N}}\right)$ where $\left\{v_{n}\right\}_{n \in \mathbb{N}}$ is a linearly dense sequence of vectors of $\mathcal{B}$ (ie $\mathcal{B}$ is the closed linear span of $\left\{v_{0}, v_{1}, \ldots\right\}$ ). If $\mathcal{B}^{\#}=\left(\mathcal{B},\left\{v_{n}\right\}_{n \in \mathbb{N}}\right)$ is a presentation of $\mathcal{B}$, then we refer to $v_{n}$ as the $n$-th distinguished vector of $\mathcal{B}^{\#}$. Thus, to define a presentation of a Banach space, it suffices to specify the distinguished vectors.

Suppose $\mathcal{B}^{\#}$ is a presentation of a Banach space $\mathcal{B}$. A rational vector of $\mathcal{B}^{\#}$ is a rational linear combination of distinguished vectors of $\mathcal{B}^{\#}$, ie a vector that can be expressed in the form $\sum_{j=0}^{n} \alpha_{j} v_{j}$ where each $\alpha_{j}$ is a rational scalar and each $v_{j}$ is a distinguished vector of $\mathcal{B}^{\#}$.

A presentation $\mathcal{B}^{\#}$ is computable if the norm function is computable on the rational vectors of $\mathcal{B}^{\#}$, ie there is an algorithm that, given a $k \in \mathbb{N}$ and a (code of) a rational vector $v$ of $\mathcal{B}^{\#}$, computes a rational number $q$ so that $\left|q-\|v\|_{\mathcal{B}}\right|<2^{-k}$. A code of such an algorithm is called an index of the presentation.

Among all presentations of a Banach space $\mathcal{B}$, one may be designated as standard; in this case, we will identify $\mathcal{B}$ with its standard presentation. The standard presentation of the field of scalars $\mathbb{F}$ is defined by declaring 1 to be the $n$-th distinguished vector for all $n$. The standard presentations of $\ell_{n}^{p}$ and $\ell^{p}$ are defined via the standard bases. The standard presentation of $L^{p}[0,1]$ is defined via the indicator functions of dyadic subintervals of $[0,1]$. The standard presentation of $L^{p}(0,1)$ is defined similarly. The standard presentation of $L^{p}\left((0,1)^{\omega}\right)$ is defined by declaring the $\langle n, k\rangle$-th distinguished 
vector to be the indicator function of $(0,1)^{n} \times I_{k} \times(0,1)^{\omega}$ where $I_{k}$ is the $k$-th dyadic subinterval of $(0,1)$. Note that these presentations are computable if $p$ is.

Fix a presentation $\mathcal{B}^{\#}$ of a Banach space $\mathcal{B}$. We say that a vector $v$ of $\mathcal{B}$ is a computable vector of $\mathcal{B}^{\#}$ if there is an algorithm that given any $k \in N$ produces a rational vector $u$ of $\mathcal{B}^{\#}$ so that $\|u-v\|_{\mathcal{B}}<2^{-k}$. A sequence $\left\{v_{n}\right\}_{n \in \mathbb{N}}$ is a computable sequence of $\mathcal{B}^{\#}$ if $v_{n}$ is a computable vector of $\mathcal{B}^{\#}$ uniformly in $n$.

We now discuss computability properties of sets of vectors. Let $\mathcal{B}^{\#}$ be a presentation of a Banach space $\mathcal{B}$, and let $X \subseteq \mathcal{B}$. We say $X$ is c.e. open if it is the union of a computable sequence of rational balls of $\mathcal{B}^{\#}$ (ie a sequence of rational balls whose centers and radii form computable sequences). We say $X$ is c.e. closed if it is closed and the set of all rational balls of $\mathcal{B}^{\#}$ that contain a point of $X$ is c.e. We say $X$ is computably compact if it is compact and if there is an algorithm that enumerates all finite sets $\left\{B_{1}, \ldots, B_{n}\right\}$ of rational balls of $\mathcal{B}^{\#}$ so that $X \subseteq \bigcup_{j} B_{j}$ and $B_{j} \cap X \neq \emptyset$ for each $j$.

We will utilize the following which is essentially Lemma 5.2.5 of [26]

Proposition 2.13 If $X \subseteq \mathbb{R}^{n}$ is bounded and c.e. closed, and if $\mathbb{R}^{n}-X$ is c.e. open, then $X$ is computably compact.

Presentations $\mathcal{B}_{0}^{\#}$ and $\mathcal{B}_{1}^{\#}$ of Banach spaces $\mathcal{B}_{0}$ and $\mathcal{B}_{1}$ respectively induce an associated class of computable maps from $\mathcal{B}_{0}^{\#}$ into $\mathcal{B}_{1}^{\#}$. Informally, for such a map $T$ it is possible to compute arbitrarily good approximations of $T(v)$ from sufficiently good approximations of $v$ for any vector $v$. More precisely, a map $T: \mathcal{B}_{0} \rightarrow \mathcal{B}_{1}$ is said to be a computable map of $\mathcal{B}_{0}^{\#}$ into $\mathcal{B}_{1}^{\#}$ if there is an algorithm $P$ with the following properties:

(1) Given a (code of a) rational ball $B_{1}$ of $\mathcal{B}_{0}^{\#}$ as input, if $P$ halts then it produces a rational ball $B_{2}$ of $\mathcal{B}_{1}^{\#}$ so that $T\left[B_{1}\right] \subseteq B_{2}$.

(2) If $U$ is a neighborhood of $T(v)$, then there is a rational ball $B_{1}$ of $\mathcal{B}_{0}^{\#}$ so that $v \in B_{1}$ and given $B_{1}, P$ produces a rational ball $B_{2} \subseteq U$.

The computability of a map can often be demonstrated by means of computable moduli of continuity. Specifically, when $T$ maps a Banach space $\mathcal{B}_{0}$ into a Banach space $\mathcal{B}_{1}$, we say $h: \mathbb{N} \rightarrow \mathbb{N}$ is a modulus of continuity for $T$ if for every $k \in \mathbb{N}$ and all vectors $v_{0}$ and $v_{1}$ of $\mathcal{B}_{0}$, if $\left\|v_{0}-v_{1}\right\|_{\mathcal{B}}<2^{-h(k)}$, then $\left\|T\left(v_{0}\right)-T\left(v_{1}\right)\right\|_{\mathcal{B}}<2^{-k}$. Now, fix presentations $\mathcal{B}_{0}^{\#}$ and $\mathcal{B}_{1}^{\#}$ of $\mathcal{B}_{0}$ and $\mathcal{B}_{1}$ respectively. It is fairly easy to show that if $T: \mathcal{B}_{0} \rightarrow \mathcal{B}_{1}$ has a computable modulus of continuity, and if $T$ maps a computable and linearly dense sequence of $\mathcal{B}_{0}^{\#}$ onto a computable sequence of $\mathcal{B}_{1}^{\#}$, then $T$ is a computable map of $\mathcal{B}_{0}^{\#}$ into $\mathcal{B}_{1}^{\#}$. It then follows that if $T$ is linear and bounded, then $T$ 
is a computable map of $\mathcal{B}_{0}^{\#}$ into $\mathcal{B}_{1}^{\#}$ if and only if $T$ maps a computable and linearly dense sequence of $\mathcal{B}_{0}^{\#}$ onto a computable sequence of $\mathcal{B}_{1}^{\#}$.

The following, which is a fairly straightforward consequence of the definitions, is folklore.

Proposition 2.14 Suppose $\mathcal{B}_{0}^{\#}$ and $\mathcal{B}_{1}^{\#}$ are computable presentations of Banach spaces, and let $T$ be a computable map of $\mathcal{B}_{0}^{\#}$ into $\mathcal{B}_{1}^{\#}$.

(1) If $U$ is a c.e. open subset of $\mathcal{B}_{1}^{\#}$, then, $T^{-1}[U]$ is a c.e. open subset of $\mathcal{B}_{0}^{\#}$.

(2) If $C$ is a c.e. closed subset of $\mathcal{B}_{0}^{\#}$, then $\overline{T[C]}$ is a c.e. closed subset of $\mathcal{B}_{1}^{\#}$.

\section{Preliminary results from computable analysis}

We first state and prove a number of fairly straightforward results on the effective theory of integration. We then prove some preliminary results on effective presentations of Banach spaces.

\subsection{Effective integration}

For the sake of computing the densities and cumulative distribution functions of stable distributions, we will need the following lemmas concerning the effective theory of integration. It will suffice to consider functions defined on the real line. Let $m$ denote Lebesgue measure on $\mathbb{R}$.

Lemma 3.1 Suppose $f: \mathbb{R}^{2} \rightarrow \mathbb{C}, \psi: \mathbb{R} \rightarrow \mathbb{R}$, and $\int_{\mathbb{R}} \psi d m$ are computable. Assume further that $|f(x, y)| \leq \psi(y)$ for all $(x, y) \in \mathbb{R}^{2}$. Then, the map

$$
x \mapsto \int_{\mathbb{R}} f(x, y) d m(y)
$$

is computable.

Proof Let $\phi(x)=\int_{\mathbb{R}} f(x, y) d m(y)$. For each $n \in \mathbb{N}$, let $g_{n}(x)=\int_{-n}^{n} f(x, y) d y$ for all $x \in R$. Thus, $g_{n}$ is computable uniformly in $n$. Since $\psi$ and $\int_{\mathbb{R}} \psi d m$ are computable, there is a computable $h: \mathbb{N} \rightarrow \mathbb{N}$ so that $\int_{|x| \geq n} \psi(x) d x<2^{-k}$ for all $k \in \mathbb{N}$. Suppose $n \in \mathbb{N}$ and $n \geq h(k)$. Then, for all $x \in \mathbb{R}$ :

$$
\begin{aligned}
\left|g_{n}(x)-\phi(x)\right| & \leq \int_{|y| \geq n}|f(x, y)| d y \\
& \leq \int_{|y| \geq n} \psi(y) d y<2^{-k}
\end{aligned}
$$


Thus, $\left\{g_{n}\right\}_{n \in \mathbb{N}}$ converges uniformly to $\phi$ with a computable modulus of convergence. Thus, $\phi$ is computable.

If $I$ is an interval, then a measurable function $f: I \rightarrow \mathbb{C}$ is said to be computably integrable if $\int_{I}|f| d m$ is a computable real.

Lemma 3.2 Suppose $\psi: \mathbb{R} \rightarrow \mathbb{R}$ is a computable and computably integrable function. Suppose $f: \mathbb{R} \rightarrow \mathbb{R}$ is a computable function for which there exists an $a \in \mathbb{N}$ so that $|f(x)| \leq \psi(x)$ whenever $|x| \geq a$. Then, $f$ is computably integrable.

Proof Let $k \in \mathbb{N}$ be given. Since $\psi$ is computably integrable, it is possible to compute an $n_{0} \in \mathbb{N}$ so that $n_{0} \geq a$ and $\int_{|x| \geq n_{0}} \psi(x) d x<2^{-(k+1)}$. Since $f$ is computable, it is possible to compute a rational number $q$ so that $\left|q-\int_{|x| \leq n_{0}}\right| f(x)|d x|<2^{-(k+1)}$.

Thus:

$$
\begin{aligned}
\left|q-\int_{\mathbb{R}}\right| f|d m| & \leq\left|q-\int_{|x| \leq n_{0}}\right| f(x)|d x|+\int_{|x| \geq n_{0}}|f(x)| d x \\
& \leq 2^{-(k+1)}+\int_{|x| \geq n_{0}} \psi(x) d x<2^{-k}
\end{aligned}
$$

Lemma 3.3 If $f: \mathbb{R} \rightarrow \mathbb{R}$ is computable and computably integrable, then $\int_{\mathbb{R}} f d m$ is computable.

Proof Since $f$ is computable, $f^{+}$and $f^{-}$are computable. Since $f$ is computably integrable, $f^{+}$and $f^{-}$are computably integrable by Lemma 3.2. It follows that $\int_{\mathbb{R}} f d m$ is computable.

Lemma 3.4 If $r$ is a computable positive real number, then $\int_{-\infty}^{\infty} \exp \left(-|t|^{r}\right) d t$ is computable.

Proof We first show that whenever $|t|$ is sufficiently large, $\exp \left(-|t|^{r}\right) \leq\left(1+t^{2}\right)^{-1}$. By L'Hospital's Rule:

$$
\begin{aligned}
\lim _{s \rightarrow \infty} \frac{\ln \left(1+s^{2}\right)}{s^{r}} & =\lim _{s \rightarrow \infty} \frac{2 s\left(1+s^{2}\right)^{-1}}{r s^{r-1}} \\
& =\lim _{s \rightarrow \infty} \frac{2}{r\left(s^{r}+s^{r-2}\right)}=0
\end{aligned}
$$

Thus, $\ln \left(1+s^{2}\right) \leq s^{r}$ whenever $s$ is sufficiently large. Therefore, $\exp \left(-s^{r}\right) \leq\left(1+s^{2}\right)^{-1}$ whenever $s$ is sufficiently large.

The computability of $\int_{-\infty}^{\infty} \exp \left(-|t|^{r}\right) d t$ now follows from Lemmas 3.2 and 3.3. 
Lemma 3.5 Suppose $f: \mathbb{R} \rightarrow \mathbb{R}$ is computable and computably integrable. Then

$$
y \mapsto \int_{-\infty}^{y} f(t) d t
$$

is computable.

Proof Set

$$
F(y)=\int_{-\infty}^{y} f(t) d t
$$

Since $f$ is computable and computably integrable, there is a computable and increasing $a: \mathbb{N} \rightarrow \mathbb{N}$ so that

$$
\int_{|t| \geq a(k)}|f(t)| d t<2^{-k}
$$

for all $k \in \mathbb{N}$.

We first show that $F$ has a computable modulus of continuity. To see this, for each $n \in \mathbb{N}$, let $F_{n}:[-n, n] \rightarrow \mathbb{R}$ be defined by

$$
F_{n}(y)=\int_{-n}^{y} f(t) d t
$$

Since $f$ is computable, $F_{n}$ is computable uniformly in $n$. Thus, there is a uniformly computable sequence $\left\{g_{n}\right\}_{n \in \mathbb{N}}$ of functions so that $g_{n}$ is a modulus of continuity for $F_{n}$ for each $n \in \mathbb{N}$. Let $h(k)=g_{a(k+1)}(k+1)$.

Since $a$ is computable and $\left\{g_{n}\right\}_{n \in \mathbb{N}}$ is uniformly computable, it follows that $h$ is computable. We now claim that $h$ is a modulus of continuity for $F$. To see this, let $y_{1}, y_{2} \in \mathbb{R}$, and suppose $\left|y_{1}-y_{2}\right|<2^{-h(k)}$. Without loss of generality, suppose $y_{1}<y_{2}$. Let $y_{1}^{\prime}=\max \left\{-a(k+1), y_{1}\right\}$, and let $y_{2}^{\prime}=\min \left\{y_{2}, a(k+1)\right\}$. Then, by inspection of cases:

$$
\left|F\left(y_{1}\right)-F\left(y_{2}\right)\right| \leq \int_{-\infty}^{a(k+1)}|f(t)| d t+\left|F\left(y_{2}^{\prime}\right)-F\left(y_{1}^{\prime}\right)\right|+\int_{a(k+1)}^{\infty}|f(t)| d t
$$

Since $\left|y_{1}-y_{2}\right|<2^{-h(k)},\left|y_{1}^{\prime}-y_{2}^{\prime}\right|<2^{-h(k)}$. Thus, $\left|F\left(y_{2}^{\prime}\right)-F\left(y_{1}^{\prime}\right)\right|<2^{-(k+1)}$ by definition of $h$. Therefore, by definition of $a,\left|F\left(y_{2}\right)-F\left(y_{1}\right)\right|<2^{-k}$.

We now show that $\{F(q)\}_{q \in \mathbb{Q}}$ is computable. Let $q \in \mathbb{Q}$, and let $k \in \mathbb{N}$. Compute $k^{\prime} \in \mathbb{N}$ so that $-a\left(k^{\prime}+1\right)<q$. Compute $q_{1} \in \mathbb{Q}$ so that

$$
\left|q_{1}-\int_{-a\left(k^{\prime}+1\right)}^{q} f(t) d t\right|<2^{-(k+1)} .
$$

Then $\left|q_{1}-F(q)\right|<2^{-k}$. Thus, $F$ is computable. 
The following more or less effectivizes a well-known trick from measure theory, and will be used for calculating absolute moments of random variables.

Lemma 3.6 Let $X$ be a random variable, and suppose $\psi(x)=P[|X| \geq x]$ for all $x \geq 0$. If $\psi$ is computable, and if there is a computable and computably integrable $h:[0, \infty) \rightarrow \mathbb{R}$ so that $\psi(x) \leq h(x)$ for all sufficiently large $x$, then $E[|X|]$ is computable.

Proof By a standard measure theory result,

$$
E[|X|]=\int_{0}^{\infty} \psi(x) d x .
$$

It now follows from Lemma 3.2 that $E[|X|]$ is computable.

\subsection{Effective presentations of Banach spaces}

Our first goal is to prove the following effective version of a well-known classical result on finite-dimensional Banach spaces.

Proposition 3.7 If $\mathcal{B}^{\#}$ is a computable presentation of a finite-dimensional Banach space, then the closed unit ball of $\mathcal{B}$ is a computably compact set of $\mathcal{B}^{\#}$.

We will need the following lemma.

Lemma 3.8 Let $n$ be a positive integer. Suppose $\mathcal{B}^{\#}$ is a computable presentation of a Banach space whose dimension is at least $n$. Then there exist $n$ rational vectors $v_{1}, \ldots, v_{n}$ of $\mathcal{B}^{\#}$ so that $\left\{v_{1}, \ldots, v_{n}\right\}$ is linearly independent.

Proof By way of contradiction suppose otherwise. Let $m$ be the largest natural number so that there exists a linearly independent set of $m$ rational vectors of $\mathcal{B}^{\#}$. Thus, $m<n$. Let $E$ be a linearly independent set of $m$ rational vectors of $\mathcal{B}^{\#}$, and let $V$ denote the linear span of $E$. Thus, since $\operatorname{dim}(\mathcal{B})>m, \mathcal{B}-V \neq \emptyset$. Since $V$ is a finite-dimensional subspace of $\mathcal{B}, V$ is closed, and so $\mathcal{B}-V$ is open. Thus, since the rational vectors of $\mathcal{B}^{\#}$ are dense in $\mathcal{B}, \mathcal{B}-V$ contains a rational vector $v$ of $\mathcal{B}^{\#}$. Hence, $E \cup\{v\}$ is a linearly independent set of rational vectors of $\mathcal{B}^{\#}$, and $E \cup\{v\}$ contains $m+1$ vectorsa contradiction. 
Proof of Proposition 3.7 Suppose $\mathcal{B}^{\#}$ is a computable presentation of a finite-dimensional Banach space, and let $n=\operatorname{dim}(B)$. Let $\mathbf{B}$ denote the closed unit ball of $\mathcal{B}$.

By Lemma 3.8 there exist $n$ rational vectors $v_{1}, \ldots, v_{n}$ of $\mathcal{B}^{\#}$ so that $\left\{v_{1}, \ldots, v_{n}\right\}$ is linearly independent. Thus, $\left\{v_{1}, \ldots, v_{n}\right\}$ is a basis of $\mathcal{B}$.

For all $\left(\alpha_{1}, \ldots, \alpha_{n}\right) \in \mathbb{F}^{n}$, let $T\left(\alpha_{1}, \ldots, \alpha_{n}\right)=\alpha_{1} v_{1}+\ldots+\alpha_{n} v_{n}$. Thus, $T$ is an isomorphism of $\mathbb{F}^{n}$ onto $\mathcal{B}$. Since $v_{1}, \ldots, v_{n}$ are computable vectors of $\mathcal{B}^{\#}$, it follows that $T$ is a computable map of $\mathbb{F}^{n}$ onto $\mathcal{B}^{\#}$.

Let $S=T^{-1}$. Since $T$ is a computable map of $\mathbb{F}^{n}$ onto $\mathcal{B}^{\#}, S$ is a computable map of $\mathcal{B}^{\#}$ onto $\mathbb{F}^{n}$. Let $V=S[\mathbf{B}]$. Since $S$ is bounded, $V$ is bounded.

We claim that $\mathbb{F}^{n}-V$ is c.e. open. To see this, note that since $T$ is bijective, $\mathbb{F}^{n}-V=S[\mathcal{B}-\mathbf{B}]$. Since $\mathcal{B}-\mathbf{B}$ is the union of all rational open balls $B(v ; r)$ of $\mathcal{B}^{\#}$ so that $\|v\|_{\mathcal{B}}-r>1, \mathcal{B}-\mathbf{B}$ is computably open. By Proposition $2.14, \mathbb{F}^{n}-V$ is computably open.

We now show $V$ is c.e. closed. By definition of $\mathbf{B}$, if $B(v ; r)$ is a rational ball of $\mathcal{B}^{\#}$, then $B(v ; r) \cap U \neq \emptyset$ if and only if $\|v\|_{\mathcal{B}}-r<1$. Thus, $\mathbf{B}$ is c.e. closed. Since $V=S[\mathbf{B}]$, it now follows from Proposition 2.14 that $V$ is c.e. closed.

It now follows from Proposition 2.13 that $V$ is computably compact.

We conclude this section by introducing the concept of a uniformly computably presentable sequence of Banach spaces. In addition, we prove a useful result for encoding a c.e. set into such a sequence.

Definition 3.9 (1) A sequence $\left(\mathcal{B}_{e}^{\#}\right)_{e \in \mathbb{N}}$ of Banach space presentations is uniformly computable if there is a computable $g: \mathbb{N} \rightarrow \mathbb{N}$ so that $g(e)$ is an index of $\mathcal{B}_{e}$ for all $e$.

(2) A sequence $\left(\mathcal{B}_{e}\right)_{e \in \mathbb{N}}$ of Banach spaces is uniformly computably presentable if there is a uniformly computable sequence $\left(\mathcal{B}_{e}^{\#}\right)_{e \in \mathbb{N}}$ of presentations.

Proposition 3.10 Suppose $\mathcal{B}_{0}^{\#}$ and $\mathcal{B}_{1}^{\#}$ are computable presentations of Banach spaces. Let $A \subseteq \mathbb{N}$ be c.e., and for all $e \in \mathbb{N}$, let:

$$
\mathcal{A}_{e}= \begin{cases}\mathcal{B}_{1} & e \notin A \\ \mathcal{B}_{0} & e \in A\end{cases}
$$

If there is a computable isometric embedding of $\mathcal{B}_{1}^{\#}$ into $\mathcal{B}_{0}^{\#}$, then $\left(\mathcal{A}_{e}\right)_{e \in \mathbb{N}}$ is uniformly computably presentable. 
Proof Fix a computable isometric embedding $T$ of $\mathcal{B}_{1}^{\#}$ into $\mathcal{B}_{0}^{\#}$. Let $v_{n}^{j}$ denote the $n$-th distinguished vector of $\mathcal{B}_{j}^{\#}$. Let $\left(A_{s}\right)_{s \in \mathbb{N}}$ be a computable enumeration of $A$. For all $e, n$, let:

$$
u_{n}^{e}=\left\{\begin{array}{cc}
v_{n}^{1} & e \notin A \\
T\left(v_{n}^{1}\right) & e \in A-A_{n} \\
v_{(n)_{0}}^{0} & e \in A_{n}
\end{array}\right.
$$

It follows that $\left(u_{n}^{e}\right)_{n \in \mathbb{N}}$ is linearly dense in $\mathcal{A}_{e}$. Let $\mathcal{A}_{e}^{\#}=\left(\mathcal{A}_{e},\left(u_{n}^{e}\right)_{n \in \mathbb{N}}\right)$.

We show $\left(\mathcal{A}_{e}^{\#}\right)_{e \in \mathbb{N}}$ is uniformly computable. By the $s-m-n$ Theorem, it suffices to show there is a computable function $f: \mathbb{N}^{2} \times \mathbb{F}_{\mathbb{Q}}^{<\omega} \rightarrow \mathbb{Q}$ so that for all $e, k \in \mathbb{N}$ and all $\alpha \in \mathbb{F}_{\mathbb{Q}}^{<\omega},\left|f(e, k, \alpha)-\left\|\sum_{j<|\alpha|} \alpha(j) u_{j}^{e}\right\|_{\mathcal{A}_{e}}\right|<2^{-k}$.

Let $\mathcal{R}_{j}$ denote the set of all rational vectors of $\mathcal{B}_{j}^{\#}$. Since $\mathcal{B}_{0}^{\#}$ is computable, there is a computable $g: \mathbb{N} \times \mathcal{R}_{0} \rightarrow \mathbb{Q}$ so that $\left|g(k, v)-\|v\|_{\mathcal{B}_{0}}\right|<2^{-k}$ for all $k \in \mathbb{N}$ and all $v \in \mathcal{R}_{0}$. Since $T$ is computable, there is a computable $h: \mathbb{N} \times \mathcal{R}_{1} \rightarrow \mathcal{R}_{0}$ so that $\left|\|T(v)\|_{\mathcal{B}_{0}}-h(k, v)\right|<2^{-k}$ for all $k \in \mathbb{N}$ and all $v \in \mathcal{R}_{1}$. Let:

$$
f(e, k, \alpha)=g\left(k+1, h\left(k+1, \sum_{j<|\alpha|} \alpha(j)\left(\left(1-\chi_{A_{j}}(e)\right) v_{j}^{1}+\chi_{A_{j}}(e) v_{j}^{0}\right)\right)\right)
$$

By definition, $f$ is computable. Let $e, k \in \mathbb{N}$, and let $\alpha \in \mathbb{F}_{\mathbb{Q}}^{<\omega}$.

Set:

$$
\begin{aligned}
\mathbf{x}_{0} & =\sum_{j<|\alpha|} \alpha(j) \chi_{A_{j}}(e) v_{j}^{0} \\
\mathbf{x}_{1} & =\sum_{j<|\alpha|} \alpha(j)\left(1-\chi_{A_{j}}(e)\right) v_{j}^{1} \\
\mathbf{x}_{2} & =h\left(k+1, \mathbf{x}_{1}\right) \\
\mathbf{y} & =\sum_{j<|\alpha|} \alpha(j) u_{j}^{e} \\
q & =g\left(k+1, \mathbf{x}_{2}+\mathbf{x}_{0}\right)
\end{aligned}
$$

Thus, $q=f(e, k, \alpha)$.

Case 1: $e \notin A$.

Thus, $\mathcal{A}_{e}=\mathcal{B}_{1}, \mathbf{x}_{0}=\mathbf{0}_{\mathcal{B}_{0}}$, and $\mathbf{y}=\mathbf{x}_{1}$. Therefore:

$$
\begin{aligned}
\left|q-\|\mathbf{y}\|_{\mathcal{A}_{e}}\right| & =\left|q-\left\|\mathbf{x}_{1}\right\|_{\mathcal{B}_{1}}\right| \\
& =\left|q-\left\|T\left(\mathbf{x}_{1}\right)\right\|_{\mathcal{B}_{0}}\right| \\
& \leq\left|q-\left\|\mathbf{x}_{2}\right\|_{\mathcal{B}_{0}}\right|+\left|\left\|\mathbf{x}_{2}\right\|_{\mathcal{B}_{0}}-\left\|T\left(\mathbf{x}_{1}\right)\right\|_{\mathcal{B}_{0}}\right| \\
& <2^{-(k+1)}+2^{-(k+1)}=2^{-k}
\end{aligned}
$$


Case 2: $e \in A$.

Thus, $\mathcal{A}_{e}=\mathcal{B}_{0}$, and $\mathbf{y}=T\left(\mathbf{x}_{1}\right)+\mathbf{x}_{0}$. We have:

$$
\left|q-\left\|T\left(\mathbf{x}_{1}\right)+\mathbf{x}_{0}\right\|_{\mathcal{B}_{0}}\right| \leq\left|q-\left\|\mathbf{x}_{2}+\mathbf{x}_{0}\right\|_{\mathcal{B}_{0}}\right|+\left|\left\|\mathbf{x}_{2}+\mathbf{x}_{0}\right\|_{\mathcal{B}_{0}}-\left\|T\left(\mathbf{x}_{1}\right)+\mathbf{x}_{0}\right\|_{\mathcal{B}_{0}}\right|
$$

By definition of $g,\left|q-\left\|\mathbf{x}_{2}+\mathbf{x}_{0}\right\|_{\mathcal{B}_{0}}\right|<2^{-(k+1)}$. By definition of $h$ :

$$
\left|\left\|\mathbf{x}_{2}+\mathbf{x}_{0}\right\|_{\mathcal{B}_{0}}-\left\|T\left(\mathbf{x}_{1}\right)+\mathbf{x}_{0}\right\|_{\mathcal{B}_{0}}\right| \leq\left\|\mathbf{x}_{2}-T\left(\mathbf{x}_{1}\right)\right\|_{\mathcal{B}_{0}}<2^{-(k+1)}
$$

So, again $\left|q-\|\mathbf{y}\|_{\mathcal{A}_{e}}\right|<2^{-k}$.

\section{Exponents larger than 2}

Theorem 4.1 If $2 \leq p<\infty$, and if there is a computable presentation of an $L^{p}$ space whose dimension is at least 2 , then $p$ is computable.

Proof We seek to deploy Krivine's Theorem (Theorem 2.3). Suppose $2 \leq p<\infty$, and let $\mathcal{B}^{\#}$ be a computable presentation of an $L^{p}$ space $\mathcal{B}$ whose dimension is at least 2 . We can assume $p$ is not an integer. Fix integers $r$, s so that $2 \leq 2(r-1)<p<2 r \leq 4 s$.

Let $n$ be a positive integer. For all $\left(x_{1}, \ldots, x_{n}\right) \in \mathbb{R}^{n}$, let $T_{n}\left(x_{1}, \ldots, x_{n}\right)=\sum_{j} x_{j}$. For all $v_{1}, \ldots, v_{n} \in \mathcal{B}$ and all $\alpha_{1}, \ldots, \alpha_{n}, q \in \mathbb{R}$, let $F_{n}\left(v_{1}, \ldots, v_{n}, \alpha_{1}, \ldots, \alpha_{n}, q\right)$ denote the expression on the left side of the main inequality in Theorem 2.3 with the exponent $p$ replaced by a trial exponent $q$. That is:

$$
F_{n}\left(v_{1}, \ldots, v_{n}, \alpha_{1}, \ldots, \alpha_{n}, q\right)=(-1)^{r} \sum_{\sigma \in\{-1,1\}^{2 s}} \sum_{\tau \in\{1, \ldots, n\}^{2 s}}\left\|\sum_{j<2 s} \sigma(j) v_{\tau(j)}\right\|_{\mathcal{B}}^{q} \cdot \prod_{j<2 s} \alpha_{\tau(j)}
$$

Thus, $F_{n}$ is a computable map from $\left(\mathcal{B}^{n}\right)^{\#} \times \mathbb{R}^{n+1}$ into $\mathbb{R}$. Let $\mathcal{U}_{n}$ denote the set of all triples $\left(B_{1}, B_{2}, I\right)$ that satisfy the following conditions.

(1) $B_{1}$ is a rational ball of $\left(\mathcal{B}^{n}\right)^{\#}$.

(2) $B_{2}$ is a rational ball of $\mathbb{R}^{n}$.

(3) $I$ is an open rational interval.

(4) $F_{n}\left[B_{1} \times B_{2} \times I\right] \subseteq(-\infty, 0)$.

(5) $B_{2} \cap T_{n}^{-1}[\{1\}] \neq \emptyset$. 
We now let:

$$
\begin{aligned}
\mathcal{V}_{n} & =\left\{I: \exists B_{1}, B_{2}\left(B_{1}, B_{2}, I\right) \in \mathcal{U}_{n}\right\} \\
V_{n} & =\bigcup \mathcal{V}_{n} \\
\mathcal{V} & =\bigcup \mathcal{V}_{n} \\
V & =\bigcup \mathcal{V}
\end{aligned}
$$

We first claim that $V \cap[2(r-1), 2 r]=[2(r-1), 2 r]-\{p\}$. To this end, we first show that $V \cap[2(r-1), 2 r] \subseteq[2(r-1), 2 r]-\{p\}$. Let $q \in V_{n} \cap[2(r-1), 2 r]$. Then, $q \in I$ for some $I \in \mathcal{V}_{n}$. Since $I \in \mathcal{V}_{n}$, there exists $B_{1}, B_{2}$ so that $\left(B_{1}, B_{2}, I\right) \in \mathcal{U}_{n}$. Thus, $F_{n}\left[B_{1} \times B_{2} \times I\right] \subseteq(-\infty, 0)$ and $B_{2} \cap T_{n}^{-1}[\{1\}] \neq \emptyset$. Let $\left(v_{1}, \ldots, v_{n}\right) \in B_{1}$, and let $\left(\alpha_{1}, \ldots, \alpha_{n}\right) \in B_{2} \cap T_{n}^{-1}[\{1\}]$. Then, $F_{n}\left(v_{1}, \ldots, v_{n}, \alpha_{1}, \ldots, \alpha_{n}, q\right)<0$ and $\sum_{j} \alpha_{j}=1$. So, by Theorem $2.3, \mathcal{B}$ does not isometrically embed into an $L^{q}$ space. Therefore, $q \neq p$, and so $V \cap[2(r-1), 2 r] \subseteq[2(r-1), 2 r]-\{p\}$.

Now, conversely, let $q \in[2(r-1), 2 r]-\{p\}$. Thus, by Theorem $2.2, \mathcal{B}$ does not isometrically embed into an $L^{q}$ space. Hence, by Theorem 2.3 , there exists a positive integer $n$, vectors $v_{1}, \ldots, v_{n} \in \mathcal{B}$, and $\left(\alpha_{1}, \ldots, \alpha_{n}\right) \in T_{n}^{-1}[\{1\}]$ so that $F_{n}\left(v_{1}, \ldots, v_{n}, \alpha_{1}, \ldots, \alpha_{n}, q\right)<0$. By continuity, there exist $\left(B_{1}, B_{2}, I\right) \in \mathcal{U}_{n}$ so that $\left(v_{1}, \ldots, v_{n}\right) \in B_{1},\left(\alpha_{1}, \ldots, \alpha_{n}\right) \in B_{2}$, and $q \in I$. Thus, $q \in V$, and so $V \cap[2(r-1), 2 r] \supseteq[2(r-1), 2 r]-\{p\}$.

We now claim that for every $k \in \mathbb{N}$, there exists $k^{\prime} \in \mathbb{N}, q \in \mathbb{Q}$, and $I_{1}, \ldots, I_{m} \in \mathcal{V}$ so that $k^{\prime} \geq k, 2(r-1)<q-2^{-k^{\prime}}<q+2^{-k^{\prime}}<2 r$, and [2(r-1), $\left.2 r\right]-$ $\left(q-2^{-k^{\prime}}, q+2^{-k^{\prime}}\right) \subseteq \bigcup_{j} I_{j}$. Let $k \in \mathbb{N}$. There exists $q \in \mathbb{Q}$ and $k^{\prime} \in \mathbb{N}$ so that $k^{\prime} \geq k$ and $p \in\left(q-2^{-k^{\prime}}, q+2^{-k^{\prime}}\right) \subseteq(2(r-1), 2 r)$. By our first claim, if $s \in\left[2(r-1), q-2^{-k^{\prime}}\right] \cup\left[q+2^{-k^{\prime}}, 2 r\right]$, then $s \in I$ for some $I \in \mathcal{V}$. By compactness, there exist $I_{1}, \ldots, I_{m} \in \mathcal{V}$ so that $\left[2(r-1), q-2^{-k^{\prime}}\right] \cup\left[q+2^{-k^{\prime}}, 2 r\right] \subseteq \bigcup_{j} I_{j}$.

We can now demonstrate $p$ is computable. Given $k \in \mathbb{N}$, wait for $k^{\prime} \in \mathbb{N}, q \in \mathbb{Q}$, $I_{1}, \ldots, I_{m} \in \mathcal{V}$ so that $k^{\prime} \geq k, 2(r-1)<q-2^{-k^{\prime}}<q+2^{-k^{\prime}}<2 r$, and $[2(r-1), 2 r]-\left(q-2^{-k^{\prime}}, q+2^{-k^{\prime}}\right) \subseteq \bigcup_{j} I_{j}$, and then output $q$. By our second claim, this search always terminates. By our first claim, $p \in\left(q-2^{-k^{\prime}}, q+2^{-k^{\prime}}\right)$ and so $|q-p|<2^{-k}$.

It follows from Lemma 6.2 of Brown, McNicholl and Melnikov [4] that if $1 \leq p<2$, and if there is a computable presentation of an $L^{p}$ space, then $p$ is right-c.e. 


\section{Detour: computable stable random variables}

Our approach to proving that exponents of Lebesgue spaces can not be uniformly computed from presentations now requires consideration of the effective content of the basic theory of stable random variables. We begin with a computable inversion theorem for characteristic functions.

Theorem 5.1 Every distribution whose characteristic function is computable and computably integrable has a computable density and a computable cumulative distribution function.

Proof Let $\phi: \mathbb{R} \rightarrow \mathbb{C}$ be computable, and suppose $\phi$ is the characteristic function of a probability measure $\mu$ on $\mathbb{R}$. Let $F$ be the distribution function of $\mu$. For all $y \in \mathbb{R}$, let

$$
f(y)=\int_{-\infty}^{\infty} e^{-i y t} \phi(t) d t
$$

By Theorem 3.3.14 of Durrett [7], $f$ is the density of $F$.

Since $\phi$ is computably integrable, it follows from Lemma 3.1 that $f$ is computable.

Since $F$ is a distribution function for a probability measure on $\mathbb{R}$, and since $f$ is the density of $F, \int_{\mathbb{R}} f d m=1$. So, by Lemma $3.5, F$ is computable.

Corollary 5.2 If the parameters of a stable distribution are computable, then its density and distribution functions are computable.

Proof Let $\mu$ be a stable distribution whose parameters are computable. Then, by Theorem 2.8, the characteristic function of $\mu$ is computable, and by Lemma 3.4 it is computably integrable. Thus, by Theorem 5.1, the density and distribution function of $\mu$ are computable.

We now address existence of computable random variables with a given stable distribution.

Theorem 5.3 If $\mu$ is a stable distribution, and if the parameters of $\mu$ are computable, then there is a computable random variable on $(0,1)$ whose distribution is $\mu$. 
Proof Let $r$ denote the stability index of $\mu$. Let $\sigma, \beta$, and $\delta$ denote the scale, skewness, and shift parameters of $\mu$ respectively. Let $F$ be the cumulative distribution function of $\mu$, and let $f$ be the density of $\mu$. Set $g(t)=\sup F^{-1}[(0, t)]$ for each $t \in(0,1)$. By Proposition 2.4, $F$ is the distribution function of $g$. It now remains to show that $g$ is computable.

Let:

$$
I=\left\{\begin{array}{cc}
(\delta, \infty) & \alpha<1 \wedge \beta=1 \\
(-\infty, \delta) & \alpha<1 \wedge \beta=-1 \\
\mathbb{R} & \text { otherwise }
\end{array}\right.
$$

By Remark 2.2.4 of Zolotarev [30], the density of $F$ is positive on $I$, and so $F$ is increasing on $I$.

Let $G=\left.F\right|_{I}$. We claim that $g=G^{-1}$. To see this, we first show that $\operatorname{ran}(G)=(0,1)$; that is $F[I]=(0,1)$. Since $F$ is a continuous distribution function, $(0,1) \subseteq \operatorname{ran}(F) \subseteq$ $[0,1]$. By Remark 2.2.4 of [30], the density of $F$ is 0 on $\mathbb{R}-I$. Thus, $F[\mathbb{R}-I] \subseteq\{0,1\}$. Therefore, $(0,1) \subseteq F[I]$. Since the density of $F$ is positive on $I, F[I]$ does not contain 0 or 1 . Thus, $F[I]=(0,1)$.

Now, let $t \in(0,1)$. It suffices to show $G^{-1}(t)=\sup F^{-1}[(0, t)]$. To this end, we first show that if $a \in F^{-1}[(0, t)]$, then $a \leq G^{-1}(t)$. Let $a \in F^{-1}[(0, t)]$, and by way of contradiction suppose $a>G^{-1}(t)$. Since $F(a)>0, a \in I$ (since the density of $F$ is zero on $\mathbb{R}-I)$. Since $F$ is increasing on $I, F(a)>F\left(G^{-1}(t)\right)=t$ - a contradiction. Thus, $a \leq G^{-1}(t)$.

Now we show $G^{-1}(t)=\sup F^{-1}[(0, t)]$. By what we have just shown, $G^{-1}(t) \geq$ $\sup F^{-1}[(0, t)]$. By way of contradiction, suppose $G^{-1}(t)>\sup F^{-1}[(0, t)]$. Suppose $G^{-1}(t)>a>\sup F^{-1}[(0, t)]$. Then, $a \in I$. So, $t>F(a)$ and thus $a \in F^{-1}[(0, t)]$ - a contradiction.

Finally, we demonstrate $g$ is computable. Since the parameters of $\mu$ are computable, by Theorem 2.8, the characteristic function of $\mu$ is computable. Thus, $F$ is computable by Theorem 5.1. Therefore, since $g=G^{-1}, g$ is computable.

We now attend to the computability of the absolute moments of stable random variables.

Theorem 5.4 Let $X$ be an $r$-stable random variable, and suppose the parameters of the distribution of $X$ are computable.

(1) If $r<2$, and if $p$ is a computable real so that $0 \leq p<r$, then $E\left[|X|^{p}\right]$ is computable. 
(2) If $r=2$, then $E\left[|X|^{p}\right]$ is computable for every nonnegative real $p$.

Proof Let $F$ be the distribution function of $X$. Let $p$ be a computable nonnegative real. For each $x \geq 0$, let $\psi(x)=m\left(\left\{t:|X(t)|^{p} \geq x\right\}\right)$.

We first claim that $\psi$ is computable. Since the parameters of the stable distribution of $X$ are computable, the characteristic function of $F$ is computable and so $F$ is computable by Theorem 5.1. Since $F$ is continuous, for each $a \in \mathbb{R}, m(\{t: X(t)=a\})=0$. Thus, $\psi(x)=1-F\left(x^{1 / p}\right)+F\left(-x^{1 / p}\right)$ for each $x \geq 0$. Since $p$ is computable, it follows that $\psi$ is computable.

We now treat the case $r=2$. We begin by showing there is a computable and computably integrable $h:[0, \infty) \rightarrow \mathbb{R}$ so that $h \geq \psi$. By Theorem 2.9, there exist a positive integer $C$ and a positive rational number $q$ so that $P[|X|>x]<$ $C \exp \left(-q x^{2}\right) x^{-1}$ for all sufficiently large $x$. Thus, for all sufficiently large $x, P\left[|X|^{p}>\right.$ $x]<C \exp \left(-q x^{-2 / p}\right) x^{-1 / p}$. However, $\exp \left(q x^{2 / p}\right)>x^{2-1 / p}$ for all sufficiently large $x$. Thus, there is a positive integer $N_{0}$ so that $\psi(x) \leq x^{-2}$ for all $x \geq N_{0}$. For all $x \geq 0$, let:

$$
h(x)=\left\{\begin{array}{cc}
1 & x<N_{0} \\
N_{0}^{2} x^{-2} & x \geq N_{0}
\end{array}\right.
$$

Thus, $h$ is computable and computably integrable, and $\psi \leq h$. It now follows from Lemma 3.6 that $E\left[|X|^{p}\right]$ is computable.

Let us now consider the case $r<2$. Assume $p<r$. Again, we proceed by showing there is a computable and computably integrable $h:[0, \infty) \rightarrow \mathbb{R}$ so that $h \geq \psi$. It follows from Theorem 2.9 that there is an integer $C>1$ so that $\psi(x) \leq C x^{-r / p}$ for all sufficiently large $x$. For all $x \geq 0$, let:

$$
h(x)=\left\{\begin{array}{cc}
C & x<1 \\
C x^{-r / p} & x \geq 1
\end{array}\right.
$$

Since $r>p, h$ is computably integrable. It again follows from Lemma 3.6 that $E\left[|X|^{p}\right]$ is computable.

We note that if $r<2$, and if $X$ is $r$-stable, then $E\left[|X|^{p}\right]=\infty$ whenever $p \geq r$.

From Theorem 5.4, we immediately obtain the following which will be used in the next section to construct an embedding.

Corollary 5.5 Suppose $p, r$ are computable reals so that $1 \leq p<r \leq 2$. Let $\mu$ be a stable distribution whose parameters are computable and whose stability index is $r$. Then, $L^{p}((0,1) ; \mathbb{R})$ contains a computable vector whose distribution is $\mu$. 
We will also require a complex version of the conclusion in Corollary 5.5.

Proposition 5.6 Suppose $p, r$ are computable reals so that $1 \leq p<r<2$. Then, $L^{p}\left((0,1)^{3} ; \mathbb{C}\right)$ contains a computable vector whose distribution is isotropic and strictly $r$-stable.

Proof Let $\tau=\cos \left(\frac{\pi r}{4}\right)^{2 / r}$. By Theorem 5.3, there is a computable $A:(0,1) \rightarrow \mathbb{R}$ so that the distribution of $A$ is symmetric $r / 2$-stable with skewness parameter 1 and scale $\tau$. By the same theorem, there is a normally distributed and computable $G:(0,1) \rightarrow \mathbb{R}$ whose mean is 0 .

For all $t_{1}, t_{2}, t_{3} \in(0,1)$, let:

$$
\begin{aligned}
\bar{A}\left(t_{1}, t_{2}, t_{3}\right) & =A\left(t_{1}\right) \\
\bar{G}_{1}\left(t_{1}, t_{2}, t_{3}\right) & =G\left(t_{2}\right) \\
\bar{G}_{2}\left(t_{1}, t_{2}, t_{3}\right) & =G\left(t_{3}\right)
\end{aligned}
$$

Then, let $X=\bar{A}^{1 / 2}\left(\bar{G}_{1}+i \bar{G}_{2}\right)$.

By Corollary 2.6.4 of [24], the distribution of $X$ is isotropic $r$-stable and symmetric. Since $X$ is a computable function from $(0,1)^{3}$ into $\mathbb{C}$, it now suffices to show that $\|X\|_{p}$ is computable. By Tonelli's Theorem,

$$
E\left[|X|^{p}\right]=E\left[|A|^{p / 2}\right] E\left[\left|\bar{G}_{1}+i \bar{G}_{2}\right|^{p}\right] .
$$

By Theorem 5.4, $E\left[|A|^{p / 2}\right]$ is computable. So, it remains to show that $E\left[\left|\bar{G}_{1}+i \bar{G}_{2}\right|^{p}\right]$ is computable. For all $x \geq 0$, let $\psi(x)=m\left(\left\{\vec{t}:\left|\bar{G}_{1}(\vec{t})+i \bar{G}_{2}(\vec{t})\right|^{p} \geq x\right\}\right)$.

We claim there are an integer $C$ and a positive rational number $q$ so that $\psi(x) \leq$ $\exp \left(-q x^{2 / p}\right)$ for all sufficiently large $x$. To demonstrate this, we first note that

$$
\begin{aligned}
\left\{\vec{t}:\left|\bar{G}_{1}(\vec{t})+i \bar{G}_{2}(\vec{t})\right| \geq x\right\} & =\left\{\vec{t}: \bar{G}_{1}(\vec{t})^{2}+i \bar{G}_{2}(\vec{t})^{2} \geq x^{2 / p}\right\} \\
& \subseteq\left\{\vec{t}: \bar{G}_{1}(\vec{t})^{2} \geq x^{2 / p} / 2\right\} \cup\left\{\vec{t}: \bar{G}_{2}(\vec{t})^{2} \geq x^{2 / p}\right\}
\end{aligned}
$$

Since $\bar{G}_{j}$ and $G$ are identically distributed, $\psi(x) \leq 2 m\left(\left\{t:|G(t)| \geq 2^{-1 / 2} x^{1 / p}\right\}\right)$. The conclusion now follows from Theorem 2.9.

Thus, by Lemma 3.6, $E\left[\left|\bar{G}_{1}+i \bar{G}_{2}\right|^{p}\right]$ is computable. Hence, $E\left[|f|^{p}\right]$ is computable. Since $p$ is computable, it follows that $\|f\|_{p}$ is computable. Since $f$ and $\|f\|_{p}$ are computable, $f$ is a computable vector of $L^{p}\left((0,1)^{3} ; \mathbb{C}\right)$. 


\section{A computable embedding result}

We now apply the material in the previous section by proving the following.

Theorem 6.1 Suppose $p, r$ are computable reals so that $1 \leq p<r \leq 2$. Then, there is a computable isometric embedding of $\ell^{r}$ into $L^{p}[0,1]$.

Theorem 6.1 is an effective version of a famous theorem due to Bretagnolle, DacuhnaCastelle and Krivine [2]. We divide the main part of the proof into the following two lemmas.

Lemma 6.2 Suppose $1 \leq p<r \leq 2$.

(1) If $\left\{g_{n}\right\}_{n \in \mathbb{N}}$ is an independent family of symmetric and strictly $r$-stable random variables in $L^{p}(\Omega ; \mathbb{R})$, then $\left\{g_{n}\right\}_{n \in \mathbb{N}}$ is $L^{r}$-formally disjointly supported.

(2) If $\left\{g_{n}\right\}_{n \in \mathbb{N}}$ is an independent family of isotropic and strictly $r$-stable complex random variables in $L^{p}(\Omega ; \mathbb{C})$, then $\left\{g_{n}\right\}_{n \in \mathbb{N}}$ is $L^{r}$-formally disjointly supported.

Proof (1): Let $M \in \mathbb{N}$, and suppose $a_{j} \in \mathbb{R}$ for each $j \leq M$.

Let:

$$
\begin{aligned}
& h_{0}=\sum_{j=0}^{M} a_{j} g_{j} \\
& h_{1}=\left(\sum_{j=0}^{M}\left|a_{j}\right|^{r}\right)^{1 / r} g_{0}
\end{aligned}
$$

Since $g_{j}$ is symmetric, $a_{j} g_{j}$ and $\left|a_{j}\right| g_{j}$ have the same distribution. Since $g_{0}$ is strictly $r$-stable and $\left\{g_{0}, \ldots, g_{M}\right\}$ is independent, it follows that $h_{0}$ and $h_{1}$ have the same distribution. Thus, $\left\|h_{0}\right\|_{p}=\left\|h_{1}\right\|_{p}$.

Therefore:

$$
\begin{aligned}
\left\|\sum_{j=0}^{M} a_{j} g_{j}\right\|_{p}^{r} & =\left\|h_{0}\right\|_{p}^{r} \\
& =\left\|h_{1}\right\|_{p}^{r} \\
& =\sum_{j=0}^{M}\left|a_{j}\right|^{r}\left\|g_{0}\right\|_{p}^{r} \\
& =\sum_{j=0}^{M}\left|a_{j}\right|^{r}\left\|g_{j}\right\|_{p}^{r}
\end{aligned}
$$

The proof of (2) is similar. 
Lemma 6.3 Suppose $1 \leq p<r \leq 2$.

(1) There is a computable sequence $\left\{g_{n}\right\}_{n \in \mathbb{N}}$ of $L^{p}\left((0,1)^{\omega} ; \mathbb{R}\right)$ so that each $g_{n}$ is symmetric strictly $r$-stable and so that $\left\{g_{n}\right\}_{n \in \mathbb{N}}$ is independent.

(2) There is a computable sequence $\left\{g_{n}\right\}_{n \in \mathbb{N}}$ of $L^{p}\left((0,1)^{\omega} ; \mathbb{C}\right)$ so that each $g_{n}$ is isotropic strictly $r$-stable and so that $\left\{g_{n}\right\}_{n \in \mathbb{N}}$ is independent.

Proof By Corollary 5.5, there is a computable vector $h_{0}$ of $L^{p}((0,1) ; \mathbb{R})$ whose distribution is symmetric strictly $r$-stable. By Proposition 5.6, there is a computable vector $h_{1}$ of $L^{p}\left((0,1)^{3} ; \mathbb{C}\right)$ that is isotropic strictly $r$-stable.

Let:

$$
g= \begin{cases}h_{0} & \mathbb{F}=\mathbb{R} \\ h_{1} & \mathbb{F}=\mathbb{C}\end{cases}
$$

For each $f \in(0,1)^{\omega}$, let:

$$
g_{n}(f)=\left\{\begin{array}{cc}
g(f(n)) & \mathbb{F}=\mathbb{R} \\
g(f(3 n), f(3 n+1), f(3 n+2)) & \mathbb{F}=\mathbb{C}
\end{array}\right.
$$

By construction, $g_{n}$ and $g$ have the same distribution, and $\left\{g_{n}\right\}_{n \in \mathbb{N}}$ is an independent sequence of random variables. Thus, if $\mathbb{F}=\mathbb{R}$, then $g_{n}$ is symmetric strictly $r$-stable, and if $\mathbb{F}=\mathbb{C}$, then $g_{n}$ is isotropic $r$-stable.

We now show $\left\{g_{n}\right\}_{n \in \mathbb{N}}$ is a computable sequence of $L^{p}\left((0,1)^{\omega}\right)$. By construction, $g_{n}$ is a computable function uniformly in $\mathbb{N}$. Since $g_{n}$ and $g$ have the same distribution, $\left\|g_{n}\right\|_{p}=\|g\|_{p}$. Since $\left\|h_{0}\right\|_{p}$ and $\left\|h_{1}\right\|_{p}$ are computable, $\|g\|_{p}$ is computable. Thus, $\left\{g_{n}\right\}_{n \in \mathbb{N}}$ is a computable sequence of $L^{p}\left((0,1)^{\omega}\right)$.

Proof of Theorem 6.1 By Lemmas 6.3 and 6.2, there is a computable sequence $\left\{g_{n}\right\}_{n \in \mathbb{N}}$ of $L^{p}\left((0,1)^{\omega}\right)$ that is $L^{r}$-formally disjointly supported and so that each $g_{n}$ is nonzero. Let $h_{n}=\left\|g_{n}\right\|_{p}^{-1} g_{n}$.

We first demonstrate that for each $f \in \ell^{r}, \sum_{n=0}^{\infty} f(n) h_{n}$ converges in the $L^{p}$-norm and $\left\|\sum_{n=0}^{\infty} f(n) h_{n}\right\|_{p}=\|f\|_{r}$. Let $f \in \ell^{r}$. If $k<m$, then since $\left\{g_{n}\right\}_{n \in \mathbb{N}}$ is $L^{r}$-formally disjointly supported,

$$
\left\|\sum_{n=k}^{m} f(n) h_{n}\right\|_{p}^{r}=\sum_{n=k}^{m}|f(n)|^{r} .
$$

Since $f \in \ell^{r}$, it follows that the partial sums of $\sum_{n=0}^{\infty} f(n) h_{n}$ form a Cauchy sequence in the $L^{p}$-norm. Since $L^{p}$ spaces are complete, $\sum_{n=0}^{\infty} f(n) h_{n}$ converges in the $L^{p}$-norm. It also follows that $\left\|\sum_{n=0}^{\infty} f(n) h_{n}\right\|_{p}=\|f\|_{r}$. 
For each $f \in \ell^{r}$, let $T_{1}(f)=\sum_{n} f(n) h_{n}$. By definition, $T_{1}$ is linear, and by what has just been shown, $T_{1}$ is an isometry. By definition, $T_{1}$ maps the standard basis of $\ell^{r}$ onto a computable sequence of $L^{p}\left((0,1)^{\omega}\right)$. Thus, $T_{1}$ is computable.

By Theorem 1.1 of [6], there is a computable isometric isomorphism of $L^{p}\left((0,1)^{\omega}\right)$ onto $L^{p}[0,1]$. Let $T=T_{2} \circ T_{1}$. Thus, $T$ is a computable isometric embedding of $\ell^{r}$ into $L^{p}[0,1]$.

\section{Computing exponents from an index with advice}

We are now ready to state and prove our main negative result.

Theorem 7.1 Suppose $I \subset[1,2]$ is an open interval. There is no computable $f: \subseteq \mathbb{N} \rightarrow \mathbb{N}$ so that for all $e \in \mathbb{N}$, if $e$ is the index of a presentation of a Lebesgue space $\mathcal{B}$ whose index lies in $I$, then $f(e)$ is an index of the exponent of $\mathcal{B}$.

Proof By way of contradiction, suppose such a function exists. Fix rational numbers $r_{1}, r_{2} \in I$ so that $r_{1}<r_{2}$. Let $k_{0}$ be the least natural number so that $r_{2}-2^{-k_{0}}>r_{1}$. Fix a computable surjection $\nu_{\mathbb{Q}}$ of $\mathbb{N}$ onto $\mathbb{Q}$. Let $S$ denote the set of all $e \in \operatorname{dom}(f)$ so that $k_{0} \in \operatorname{dom}\left(\phi_{f(e)}\right)$ and $\left|\nu_{\mathbb{Q}}\left(\phi_{f(e)}\left(k_{0}\right)\right)-r_{2}\right|<2^{-k_{0}}$. Thus, $S$ is c.e.

Let

$$
\mathcal{B}_{e}=\left\{\begin{array}{cc}
L^{r_{1}}[0,1] & e \in S \\
\ell^{r_{2}} & e \notin S
\end{array}\right.
$$

By Proposition $3.10,\left(\mathcal{B}_{e}\right)_{e \in \mathbb{N}}$ is uniformly computably presentable. Thus, there is a computable $g: \mathbb{N} \rightarrow \mathbb{N}$ so that $g(e)$ indexes a presentation of $\mathcal{B}_{e}$ for all $e \in N$. By the Recursion Theorem, there is an $e_{0} \in \mathbb{N}$ so that $\phi_{g\left(e_{0}\right)}=\phi_{e_{0}}$. Thus, $e_{0}$ indexes $\mathcal{B}_{e_{0}}$. Therefore, $f\left(e_{0}\right)$ is defined. Since $e_{0} \in \operatorname{dom}(f)$, if $e_{0} \notin S$, then $f\left(e_{0}\right)$ does not index $r_{2}$. Thus, $\mathcal{B}_{e_{0}}=L^{r_{1}}[0,1]$, and $e_{0} \in S$. But, then $e_{0}$ does not index $r_{1}$ - a contradiction.

\section{The finite-dimensional case}

Although there is no uniform solution of our problem, we can take some comfort in the fact that in the finite-dimensional case, the exponent of a Lebesgue space can be computed from one of its presentations.

Theorem 8.1 If $n \geq 2$, and if $\ell_{n}^{p}$ is computably presentable where $1 \leq p<\infty$, then $p$ is computable. 
Proof We can assume $p>1$. Let $\left(\ell_{n}^{p}\right)^{\#}$ be a computable presentation of $\ell_{n}^{p}$.

By Lemma 3.8, there exist $n$ computable unit vectors $v_{1}, \ldots, v_{n}$ of $\left(\ell_{n}^{p}\right)^{\#}$ so that $\left\{v_{1}, \ldots, v_{n}\right\}$ is linearly independent.

Let $\mathcal{I}$ denote the set of all rational open intervals $I \subseteq(1, \infty)$ for which there exist rational open balls $B_{1}, \ldots, B_{k}$ of $\ell_{n^{2}}^{\infty}$ that satisfy the following two conditions.

(1) $\left\{B_{1}, \ldots, B_{k}\right\}$ covers the closed unit ball of $\ell_{n^{2}}^{\infty}$.

(2) For each $j \in\{1, \ldots, k\}$, there exist rational scalars $\alpha_{1}^{(j)}, \ldots, \alpha_{n}^{(j)}$ so that

$$
\left\|\sum_{t=1}^{n} \alpha_{t}^{(j)} v_{t}\right\|_{p} \neq\left\|\sum_{t=1}^{n} \alpha_{t}^{(j)} u_{t}\right\|_{r}
$$

for all $r \in I$ and all $u_{1}, \ldots, u_{n} \in \ell_{n}^{\infty}$ so that $u_{j} \in B_{j}$ for each $j$.

We first show that $\bigcup \mathcal{I}=(1, \infty)-\{p\}$. Suppose $r \in \bigcup \mathcal{I}$. Then, there exists $I \in \mathcal{I}$ so that $r \in I$. Thus, by definition of $\mathcal{I}, r \in(1, \infty)$. Let $B_{1}, \ldots, B_{k}$ and $\left\{\alpha_{t}^{(j)}\right\}_{t, j}$ witness that $r \in I$. Since $\left\|v_{j}\right\|_{\infty} \leq\left\|v_{j}\right\|_{p},\left\|v_{j}\right\|_{\infty} \leq 1$. Thus, by $(1),\left(v_{1}, \ldots, v_{n}\right) \in B_{j}$ for some $j$. Therefore, by (2):

$$
\left\|\sum_{t=1}^{n} \alpha_{t}^{(j)} v_{t}\right\|_{p} \neq\left\|\sum_{t=1}^{n} \alpha_{t}^{(j)} v_{t}\right\|_{r}
$$

Thus, $r \neq p$.

Conversely, suppose $r \in(1, \infty)-\{p\}$. Let $\mathbf{B}$ denote the closed unit ball of $\ell_{n^{2}}^{\infty}$. Note that $u \in \mathbf{B}$ if and only if there exist $u_{1}, \ldots, u_{n} \in \ell_{n}^{\infty}$ so that $u=\left(u_{1}, \ldots, u_{n}\right)$.

We claim that for all $\mathbf{x} \in \mathbf{B}$, there exist a rational ball $\mathbf{B}_{\mathbf{x}}$ of $\ell_{n^{2}}^{\infty}$, rational scalars $\beta_{\mathbf{x}, 1}, \ldots, \beta_{\mathbf{x}, n}$, and a rational open interval $I_{\mathbf{x}} \subseteq(1, \infty)$ so that $\mathbf{x} \in \mathbf{B}_{\mathbf{x}}, r \in I_{\mathbf{x}}$, and

$$
\left\|\sum_{j} \beta_{\mathbf{x}, j} v_{j}\right\|_{p} \neq\left\|\sum_{j} \beta_{\mathbf{x}, j} u_{j}\right\|_{r^{\prime}}
$$

for all $r^{\prime} \in I_{\mathbf{x}}$ and all $u_{1}, \ldots, u_{n} \in \ell_{n}^{\infty}$ so that $\left(u_{1}, \ldots, u_{n}\right) \in \mathbf{B}$.

Let $\mathbf{x} \in \mathbf{B}$. There exist $\mathbf{x}_{1}, \ldots, \mathbf{x}_{n} \in \ell_{n}^{\infty}$ so that $\mathbf{x}=\left(\mathbf{x}_{1}, \ldots, \mathbf{x}_{n}\right)$. Since $\left\{v_{1}, \ldots, v_{n}\right\}$ is linearly independent, there is a unique linear function $T: \mathbb{F}^{n} \rightarrow \mathbb{F}^{n}$ so that $T\left(v_{j}\right)=\mathbf{x}_{j}$ for each $j \in\{1, \ldots, n\}$. Since $r \neq p, T$ is not an isometry of $\ell_{n}^{r}$ into $\ell_{n}^{p}$ (otherwise, since $T$ is an endomorphism, $T$ is onto). However, since $T$ is linear, $T$ is a continuous map of $\mathbb{F}^{n}$ into $\ell_{n}^{p}$. Thus, there exist rational scalars $\beta_{\mathbf{x}, 1}, \ldots, \beta_{\mathbf{x}, n}$ so that $\left\|T\left(\sum_{j} \beta_{\mathbf{x}, j} v_{j}\right)\right\|_{p} \neq\left\|\sum_{j} \beta_{\mathbf{x}, j} v_{j}\right\|_{r}$. That is, $\left\|\sum_{j} \beta_{\mathbf{x}, j} \mathbf{x}_{j}\right\|_{p} \neq\left\|\sum_{j} \beta_{\mathbf{x}, j} v_{j}\right\|_{r}$. By continuity again, there is a rational interval $I_{\mathbf{x}} \subseteq(1, \infty)$ and a rational open ball $\mathbf{B}_{\mathbf{x}}$ as required. 
Since $\mathbf{B}$ is compact, there exist $\mathbf{x}^{(1)}, \ldots, \mathbf{x}^{(k)} \in \mathbf{B}$ so that $\mathbf{B} \subseteq \bigcup_{j} \mathbf{B}_{\mathbf{x}^{(j)}}$.

Let:

$$
\begin{aligned}
I & =\bigcap_{j} I_{\mathbf{x}^{(j)}} \\
B_{j} & =\mathbf{B}_{x^{(j)}} \\
\alpha_{t}^{(j)} & =\beta_{\mathbf{x}^{(j)}, t}
\end{aligned}
$$

Then, $I, B_{1}, \ldots, B_{k}$, and $\left\{\alpha_{t}^{(j)}\right\}_{t, j}$ satisfy (1) and (2). Thus, $I \in \mathcal{I}$ and so $r \in \bigcup \mathcal{I}$. This completes the demonstration that $\bigcup \mathcal{I}=(1, \infty)-\{p\}$.

We now show that $\mathcal{I}$ is c.e. By Proposition 3.7, condition (1) is $\Sigma_{1}^{0}$. Since $\left(\ell_{n}^{p}\right)^{\#}$ is a computable presentation and each $v_{j}$ is a rational vector of this presentation, it follows that condition (2) is $\Sigma_{1}^{0}$. Thus, $\mathcal{I}$ is c.e.

We can now show $p$ is computable. Fix an integer $N_{0}>p$. Given $k \in \mathbb{N}$, search for a rational number $q$ and rational open intervals $I_{1}, \ldots, I_{k} \in \mathcal{I}$ so that $[q-$ $\left.2^{-(k+1)}, q+2^{-(k+1)}\right] \subseteq \bigcup_{i} I_{j}$, and output $q$. Since $\bigcup \mathcal{I}=(1, \infty)-\{p\}$, it follows that $p \in\left[q-2^{-(k+1)}, q+2^{-(k+1)}\right]$ and so $|p-q|<2^{-k}$.

\section{Conclusion}

We began our deliberations with a very natural question: "Does the computable presentability of a Lebesgue space imply the computability of its exponent?" We immediately see that the answer is negative for Lebesgue spaces of dimension 1. For Lebesgue spaces of dimension at least 2, the "obvious" answer is 'yes', but this turns out to be difficult to prove even in the particular cases considered here. However, even though the consideration of these cases turns out to be unexpectedly difficult, their resolution and the demonstration of the non-uniformity result led to a number of surprising connections with functional analysis and probability.

The one remaining case is that of infinite-dimensional spaces whose exponent is smaller than 2. The best result so far for this case is that the exponent must be right-c.e. [4]. This result is obtained via the modulus of uniform convexity. There are a number of other moduli for describing geometric properties of Banach spaces, but these all lead to the exact same conclusion as does the consideration of $p$-types. On the basis of these observations and our non-uniformity result, we conjecture that if $p$ is a right-c.e. real so that $1 \leq p \leq 2$, then every infinite-dimensional and separable $L^{p}$ space is computably presentable. 


\section{Acknowledgement}

The author thanks the anonymous referee for several helpful comments and encouraging remarks.

\section{References}

[1] S Banach, Theory of linear operations, volume 38 of North-Holland Mathematical Library, North-Holland Publishing Co., Amsterdam (1987). Translated from the French by F. Jellett, with comments by A. Pełczyński and Cz. Bessaga; https://doi.org/10. 1016/s0924-6509(08)70001

[2] J Bretagnolle, D Dacunha-Castelle, J-L Krivine, Lois stables et espaces $L^{p}$, Ann. Inst. H. Poincaré Sect. B (N.S.) 2 (1965/1966) 231-259; https://doi.org/10.1007/bfb0061106

[3] T Brown, T H McNicholl, Analytic computable structure theory and $L^{p}$-spaces part 2, Arch. Math. Logic 59 (2020) 427-443; https://doi.org/10.1007/s00153-019-00697-4

[4] T Brown, T H McNicholl, A G Melnikov, On the complexity of classifying Lebesgue spaces, J. Symb. Log. (2020, to appear); arXiv:1906.12209; https://doi.org/ 10.1017/js1.2020.63

[5] P Cembranos, J Mendoza, Banach spaces of vector-valued functions, volume 1676 of Lecture Notes in Mathematics, Springer-Verlag, Berlin (1997); https://doi.org/10. 1007/BFb0096765

[6] J Clanin, T H McNicholl, D M Stull, Analytic computable structure theory and $L^{p}$ spaces, Fund. Math. 244 (2019) 255-285; https://doi.org/10.4064/fm448-5-2018

[7] R Durrett, Probability: theory and examples, volume 31 of Cambridge Series in Statistical and Probabilistic Mathematics, fourth edition, Cambridge University Press, Cambridge (2010); https://doi.org/10.1017/CBO9780511779398

[8] W Feller, An introduction to probability theory and its applications. Vol. I, Second edition, John Wiley and Sons, Inc., New York; Chapman and Hall, Ltd., London (1957); https://doi.org/10.1016/s0924-6509(08)70001

[9] W Feller, An introduction to probability theory and its applications. Vol. II, Second edition, John Wiley \& Sons, Inc., New York-London-Sydney (1971)

[10] O Hanner, On the uniform convexity of $L^{p}$ and $l^{p}$, Ark. Mat. 3 (1956) 239-244; https://doi.org/10.1007/BF02589410

[11] J Holtsmark, Über die Verbreiterung von Spektrallinien, Annalen der Physik 363 (1919) 577-630; https://doi.org/10.1002/andp.19193630702

[12] J-L Krivine, Plongement des espaces normés dans les $L^{p}$ pour $p>2$, C. R. Acad. Sci. Paris 261 (1965) 4307-4310; https://doi.org/10.1007/BF02589410 
[13] D Kunkle, Type-2 computability on spaces of integrable functions, MLQ Math. Log. Q. 50 (2004) 417-430; https://doi.org/10.1002/malq.200310109

[14] J Lamperti, On the isometries of certain function-spaces, Pacific J. Math. 8 (1958) 459-466; https://doi.org/10.2140/pjm.1958.8.459

[15] P Lévy, Calcul des probabilités, Gauthier-Villars, Paris (1925); https://doi.org/10.1002/ malq.200310109

[16] P Lévy, Théorie de l'addition des variables alétoires, 2 edition, Gauthier-Villars (1954)

[17] TH McNicholl, Computable copies of $\ell^{p}$, Computability 6 (2017) 391 - 408; https://doi.org/10.3233/com-160065

[18] T McNicholl, Continuous logic and embeddings of Lebesgue spaces, Arch. Math. Logic (2020, to appear); arXiv:1805.11561; https://doi.org/10.1007/s00153-020-00734-7

[19] A G Melnikov, Computably isometric spaces, J. Symbolic Logic 78 (2013) 1055-1085; https://doi.org/10.2178/js1.7804030

[20] A G Melnikov, KM Ng, Computable structures and operations on the space of continuous functions, Fund. Math. 233 (2016) 101-141; https://doi.org/10.4064/fm36$12-2015$

[21] A G Melnikov, A Nies, The classification problem for compact computable metric spaces, from: "The nature of computation", Lecture Notes in Comput. Sci. 7921, Springer, Heidelberg (2013) 320-328; https://doi.org/10.1007/978-3-642-39053-1_37

[22] R E A C Paley, Some theorems on abstract spaces, Bull. Amer. Math. Soc. 42 (1936) 235-240; https://doi.org/10.1090/S0002-9904-1936-06277-4

[23] M B Pour-El, J I Richards, Computability in analysis and physics, Perspectives in Mathematical Logic, Springer-Verlag, Berlin (1989); https://doi.org/10.1007/978-3-66221717-7

[24] G Samorodnitsky, MS Taqqu, Stable Non-Gaussian Random Processes; Stochastic Models with Infinite Variance: Stochastic Modeling (1st ed.) Taylor \& Francis, New York (1994); https://doi.org/10.1201/9780203738818

[25] K Uchiyama, Asymptotically stable random walks of index $1<\alpha<2$ killed on a finite set, Stochastic Process. Appl. 129 (2019) 5151-5199; https://doi.org/10.1016/j. spa.2019.02.006

[26] K Weihrauch, Computable analysis, Texts in Theoretical Computer Science. An EATCS Series, Springer-Verlag, Berlin (2000); https://doi.org/10.1007/978-3-642-56999-9

[27] L Xu, Approximation of stable law in Wasserstein-1 distance by Stein's method, Ann. Appl. Probab. 29 (2019) 458-504; https://doi.org/10.1214/18-AAP1424

[28] G Yu, C Li, J Zhang, A new statistical modeling and detection method for rolling element bearing faults based on $\alpha$-stable distribution, Mechanical Systems and Signal Processing 41 (2013) 155 - 175; https://doi.org/https://doi.org/10.1016/j.ymssp.2013.08.015 
[29] N Zhong, B-Y Zhang, $L^{p}$-computability, MLQ Math. Log. Q. 45 (1999) 449-456; https://doi.org/10.1002/malq.19990450403

[30] V M Zolotarev, One-dimensional stable distributions, volume 65 of Translations of Mathematical Monographs, American Mathematical Society, Providence, RI (1986). Translated from the Russian by H. H. McFaden, translation edited by Ben Silver; https://doi.org/10.1090/mmono/065

Department of Mathematics, Iowa State University, Ames, Iowa 50011

mcnichol@iastate.edu

Received: 5 March 2020 Revised: 15 October 2020 